\title{
Gastric Contractility Modulation in the Management of Type 2 Diabetes
}

\author{
a report by \\ Harold E Lebovitz, MD, FACE \\ Professor of Medicine, Division of Endocrinology, The State University of New York Health Science Center, Brooklyn
}

DOI: $10.17925 /$ USE.2007.00.2.39

Electrical stimulation of the gastric antrum with gastric contractility modulation (GCM) by the TANTALUS II system is a new modality that shows great promise as a means of treating overweight and obese type 2 diabetic patients. Preliminary studies in several small type 2 diabetic populations in patients who are inadequately controlled with oral antidiabetic agents have shown decreases in glycated hemoglobin $\left(\mathrm{HbA}_{1 \mathrm{c}}\right)$ ranging from 1.1 to $1.9 \%$ after three months of treatment. Modest weight loss is an added benefit.

The role of the gastrointestinal tract in the regulation of energy balance is now well recognized, and this knowledge has spawned the development of several new pharmacological agents and surgical procedures. Incretin mimetic agents such as exenatide and dipeptidyl peptidase (DPP-4) inhibitors are widely used to treat patients with type 2 diabetes. 'Gastric bypass surgery has a remarkable effect in acutely ameliorating hyperglycemia in type 2 diabetic patients even before there is significant weight loss. ${ }^{2}$ Gastrointestinal peptide hormones, such as amylin, glucagonlike peptide 1(GLP-1), gastric inhibitory polypeptide (GIP), and peptide YY (PYY), increase satiety through central nervous system (CNS)-mediated pathways. ${ }^{3.4}$ The gastric hormone ghrelin, in contrast, stimulates hunger through a different CNS-mediated pathway. ${ }^{3}$ GLP-1 and GIP have multiple stimulatory effects on pancreatic beta-cell function and insulin secretion. ${ }^{\prime}$ Amylin and GLP-1 inhibit glucagon secretion from pancreatic alpha cells. ${ }^{1,4}$

The stomach plays an integral part in this gastrointestinal regulation of energy balance. Dilatation of the stomach by food ingestion sends afferent signals through the vagus nerve to the CNS and increases satiety, thus regulating food intake..$^{5-8}$ Because of the many neurohormonal functions of the stomach and their modification by food ingestion, modifying the function of this organ has been hypothesized to be a potential mechanism for treating overweight and obese type 2 diabetic patients.

Many aspects of cell function are controlled by intrinsic or extrinsic electrical activity. In the normal physiological state, an action potential is generated and then causes the cellular response. When electrical signals are applied externally, they increase the rate at which the response occurs, which is called electrical pacing. However, if the electrical signal is applied during the refractory period of the normal action potential, it cannot increase the response rate. However, it has been shown in several studies to change the biochemistry of the cell, which leads to greatly increased responses to the next normal action potential. ${ }^{9}$ This phenomenon is called electroceutical stimulation and is illustrated in Figure 1. When such a signal is applied to the gastric antral muscle it causes the contraction force to be increased several-fold. The antral muscle reacts to this increased contraction force as it would to stomach dilatation with food, and increases the afferent signaling to the CNS. ${ }^{10}$ The CNS response is to increase satiety and perhaps influence CNS pathways, which are known to regulate hepatic glucose metabolism. ${ }^{11,12}$ The signals applied to the stomach in this manner are referred to as GCM signals.

\section{TANTALUS II System}

The TANTALUS II system was developed to stimulate the gastric antral muscle electroceutically immediately following the entrance of food into the stomach. Figure 2 shows the components of the TANTALUS II system. Three pairs of bipolar electrodes are implanted by laparoscopic techniques into the antrum and fundus subserosa. Wires attached to the electrodes are brought out and connected to the pulse generator, which is placed in a pocket created in the subcutaneous abdominal fat. The pulse generator is rechargeable and is functional for several years. The electrical signal and antral function can be monitored and modified, if needed, by the external logger, which is placed over the pulse generator and displays the characteristics of the electrical signaling and the gastric responses when the patient is given a test meal during follow-up visits. The external wand is used if desired to activate the pulse generator at the start of each major meal.

Eating is detected in two ways. Fundal expansion as the food enters the stomach causes a decrease in impedance. Antral pulses slow when food enters the stomach and this is detected by the antral electrode. TANTALUS $\|$ is activated by the patient using the wand prior to initiating each major meal. If the patient fails to activate the pulse generator using the wand, the automatic eating detection will activate the pulse generator as the food is detected when it enters the stomach. GCM activation lasts for a pre-defined period (ordinarily 90 minutes). Because of GCM activation, vagal afferent signals are initiated earlier and are more intense than those that occur with ordinary meal ingestion.

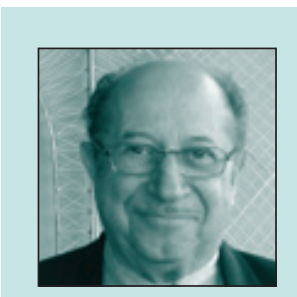

Harold E Lebovitz, MD, FACE, is Professor of Medicine in the Division of Endocrinology at The State University of New York Health Science Center, Brooklyn. Dr Lebovitz is an internationally recognized authority in the field of diabetes. He is author or co-author of more than 200 peer-reviewed publications and 100 chapters or books, and has served on numerous boards and advisory panels. He serves on the Board of Directors of the American Association of Clinical Endocrinologists (AACE) and chairs the Program Committee for the 2008 Annual Scientific Program of the American Diabetes Association (ADA). He began working at The State University of New York Health Science Center in 1982 as Professor of Medicine, Chief of the Section of Endocrinology and Diabetes, and Director of the National Institutes of Health (NIH)-sponsored Clinical Research Center. He remained as Chief of the Section of Endocrinology and Diabetes throughout the spring of 1999. He was Director of the Division of Endocrinology at Duke University from 1964 to 1982. Dr Lebovitz received his BSC and MD from the University of Pittsburgh.

E: hlebovitz@hotmail.com 
Figure 1: The Principle of Electroceutical Stimulation of the Gastric Antral Muscle

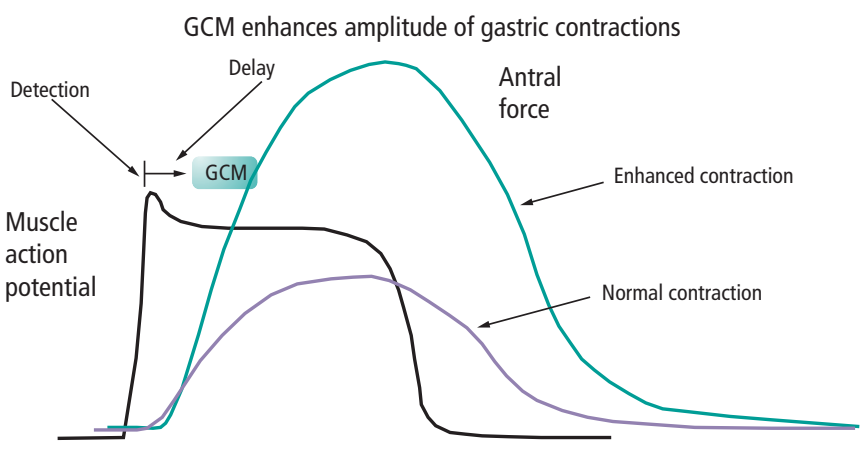

$\mathrm{GCM}=$ gastric contractility modulation

The TANTALUS II system is programmed to deliver the electrical signal during the refractory period of the normal action potential. The effect is to increase the amplitude of the contraction force without changing the rate of contraction. This increase in contraction force increases afferent signaling to the central nervous system (CNS) through the vagus and perhaps other nerves. Non-excitatory stimulation is based on the impulse technology.

Figure 2: Components of the TANTALUS II System

\section{The TANTALUS II System}

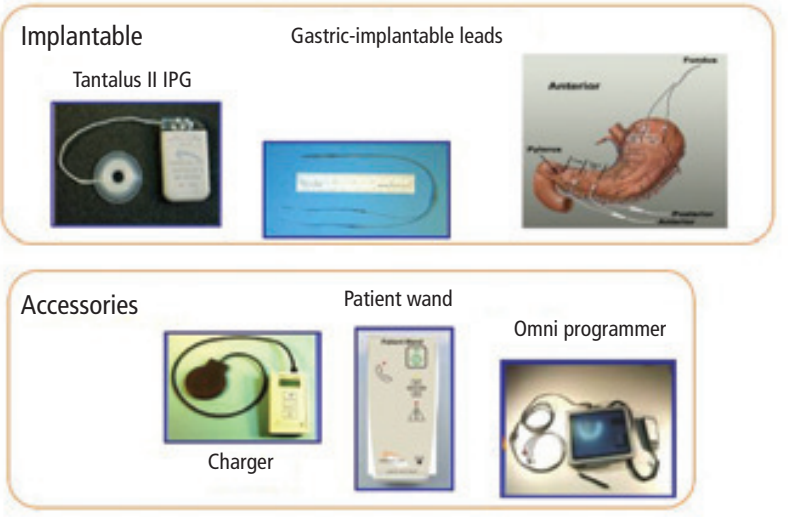

Eating detection is a crucial element of the TANTALUS II system. Rapid and effective detection of food intake is necessary to cause immediate gastric responses and facilitate patient compliance. Food intake is detected both by changes in fundal impedance and slowing of intrinsic antral electrical activity. The threshold size of the meal to be detected is important as the pulse generator has a refractory period. The number of true detections must greatly exceed the number of false detections, and the number of meals not detected must be a small fraction. The current device is able to detect $80 \%$ of meals $\geq 250$ kcals with very few missed detections or false detections.

\section{Preliminary Results}

Exploratory studies have been carried out in both morbidly obese non-diabetic patients and overweight or obese type 2 diabetic patients in India, Europe, and the US. In an open-label, non-randomized study in Europe, 12 morbidly obese subjects with a mean body mass index (BMI) of $43.2 \pm 2.7 \mathrm{~kg} / \mathrm{m}^{2}$ and a mean bodyweight of $128.8 \pm 5.2 \mathrm{~kg}$ were implanted by a laparoscopic technique. ${ }^{13}$ After 14 weeks of GCM stimulation the mean bodyweight decreased to $119.9 \pm 5.9 \mathrm{~kg}(p<0.05)$. Nine of the patients continued treatment for 46 weeks and their mean bodyweight decreased to $112.4 \pm 3.8 \mathrm{~kg}$ (a $12.7 \%$ decrease in bodyweight).

The initial studies in type 2 diabetic patients were performed in India. Five patients inadequately controlled on metformin and/or sulfonylureas with a mean BMl of $27.5 \pm 4.4 \mathrm{~kg} / \mathrm{m}^{2}$ and a mean $\mathrm{HbA}_{1 \mathrm{c}}$ of $7.7 \pm 0.4 \%$ were implanted with the first version of the TANTALUS II system, which was not rechargeable, and the pulse generator was manually activated before each of the three major meals. The patients were treated for 10-14 weeks, after which time the device and electrodes were removed. The mean decrease in bodyweight was $9 \%$, and the mean decrease in $\mathrm{HbA}_{1 \mathrm{c}}$ was $1.9 \%$. Several months after the removal of the device the $\mathrm{HbA}_{1 \mathrm{c}}$ values had returned to the pre-treatment levels and the weight was regained.

Subsequent to this proof-of-principle study, TANTALUS treatment of overweight and obese type 2 diabetic patients inadequately controlled on oral antidiabetic medications has been initiated in Austria, Germany, the US, Israel, and France. To date, ongoing studies in these centers show decreases in mean $\mathrm{HbA}_{1 \mathrm{c}}$ of $1.0-1.2 \%$ and in bodyweight of $3-5 \%$ after three months of treatment. Figure 3 depicts the changes in $\mathrm{HbA}_{1 \mathrm{c}}$ in a subset of 29 such patients. The mean $\mathrm{HbA}_{1 \mathrm{c}}$ decreased from 8.1 to $6.95 \%$. Nine patients (31\%) reduced their $\mathrm{HbA}_{1 \mathrm{c}}$ to $6.5 \%$, and $16(55 \%)$ attained treatment $\mathrm{HbA}_{1 \mathrm{c}} \leq 7.0 \%$.

\section{Discussion}

The concept of applying electrical signals to cells during the refractory period of their normal action potentials to enhance function evolved during several years of study with myocardial cells in vitro. ${ }^{9}$ This concept was extended in vivo and resulted in the development of a device to treat chronic congestive heart failure. Applying cardiac contractile modulation (CCM) improves ventricular function and re-establishes gene expression from that of a failing heart to that of a more normal functioning heart. CCM reverses the gene expression even in areas of the myocardium distant from the site of CCM stimulation. ${ }^{14,15}$ CCM is associated with phosphorylation of a unique cardiac protein, and this may be the signal for the other changes observed.

The preliminary studies with GCM modulation in overweight and obese type 2 diabetic patients raise many theoretical and practical questions. All of the studies to date have been non-randomized and open-label with no placebo-controlled group. Beneficial effects are reversed in patients who have the device removed, which is the strongest argument to suggest that the observed effect is truly an effect of the GCM stimulation. A large pivotal study that will properly address this issue is under way in the US. Several hundred patients are being entered into a randomized, blinded study in which all patients are being implanted with the TANTALUS system. Half of the patients will be delivered CGM by the pulse generator for the first six months, and all patients will receive GCM in months seven to 12 . Little is known about the durability of the GCM effects, as the rechargeable device has only recently become available. Previously, chronic therapy required replacement of the pulse generator in the subcutaneous pocket under local anesthesia every three to four months. In the few patients who have had one year of treatment or longer with multiple pulse generator replacements, the beneficial effects appear to be sustained.

The mechanism of action by which GCM causes weight loss appears to be through increasing satiety. The device creates more immediate and more intense than normal satiety signals sent from the antral muscle to the brain, 
presumably via vagal afferent impulses. The satiety centers stimulated are probably the area postrema and the nucleus accumbens, which are also the sites of action of the gastrointestinal hormones such as amylin, GLP-1, and GIP, which increase satiety. ${ }^{3}$

The mechanism by which glycemic control is improved is speculative, since definitive studies are just beginning. Weight loss itself accounts for a very small proportion of the decrease in $\mathrm{HbA}_{1 \mathrm{c}}$ (approximately $16 \%$ ) as assessed by correlation calculations. Dissociation between weight loss and decreases in $\mathrm{HbA}_{1 \mathrm{c}}$ are commonly seen, with some patients having much weight loss and minimal decreases in $\mathrm{HbA}_{1 \mathrm{c}}$, and others having little or no weight loss and striking decreases in $\mathrm{HbA}_{1 \mathrm{c}}$. The likely weight-independent possibilities for improving glycemic control include activation of CNS pathways that regulate hepatic glucose production, 11,12,16,17 alteration in the secretion of specific gastrointestinal hormones_-such as ghrelin, GLP-1, or amylinand specific decrease in visceral (omental) adipose tissue, resulting in reduction in the components of the metabolic syndrome.

The TANTALUS GCM system differs significantly from other gastric electrical stimulatory devices that have been developed and tested previously. ${ }^{18-20}$ The other devices use electrical pacing rather than electroceutical stimulation. They stimulate the stomach continuously rather than only post-prandially, and they do not have an eating detection component. The electrode is placed on the upper third of the lesser curvature of the stomach, and is connected by wire to the electric pulsator, which is placed in a pocket in the subcutaneous fat. The characteristics of the signals generated are quite different.

Variable degrees of weight loss have been reported after six months of therapy in a small number of morbidly obese individuals. The best results reported were a decrease in excess weight of $21.0 \pm 3.5 \%$. Measurements of the effects of the gastric pacing device on gastrointestinal hormone secretions have given inconsistent results with ghrelin ${ }^{18,21}$ and shown suggestive decreases in GLP-1.22 It is clear that the electrical pacing devices and the TANTALUS II GCM system are totally different in their design and function.

The use of GCM in the treatment of patients with type 2 diabetes appears to be helpful as a supplement to inadequate oral-agent antidiabetic therapy. The ongoing studies suggest that this population can achieve significant benefits
Figure 3: The Effect of Three Months of Treatment with the TANTALUS II System

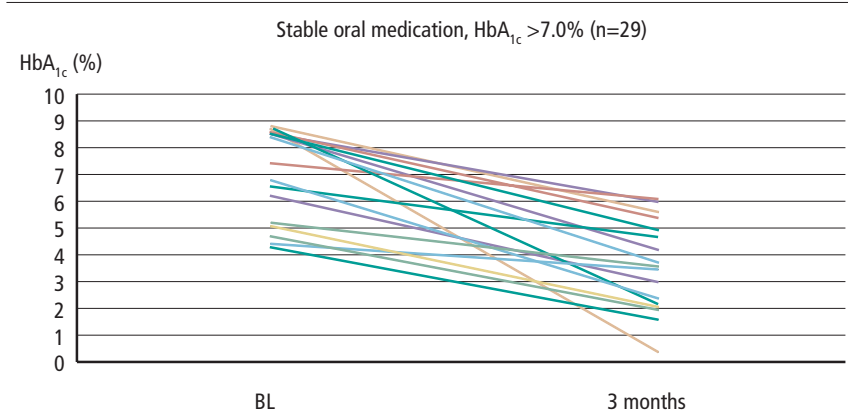

9 subjects ( $31 \%$ ) reached $\mathrm{HbA} \leq 6.5 \%$

16 subjects ( $55 \%$ ) reached $\mathrm{HbA}_{1 \mathrm{c}} \leqq 7.0 \%$

22 subjects $(76 \%)$ reached $\mathrm{HbA}_{1 \mathrm{c}} \leqq 7.5 \%$

Effects on type 2 diabetic patients inadequately controlled on combinations of oral antihypoglycemic agents. Mean baseline glycated hemoglobin $(\mathrm{HbA} 1 \mathrm{~d})=8.1 \%$. Mean $\mathrm{HbA} 1 \mathrm{c}$ after three months of stimulation $=6.95 \%$.

from the TANTALUS system. Major advantages of GCM therapy are its lack of significant metabolic side effects, such as hypoglycemia, edema, and weight gain. Since its mode of action is through normal physiological pathways, it is unlikely to be associated with deleterious cardiovascular or bone abnormalities. Addition of GCM therapy to type 2 diabetic patients inadequately controlled on oral antidiabetic agents has not only significantly decreased $\mathrm{HbA}_{1 \mathrm{c}}$ but, in a number of patients, has allowed reduction of oral agents. Little is known about its effects when used in conjunction with insulin therapy or its effects compared with insulin when added to oral agent failures, as those studies have not yet been initiated.

The side effects of TANTALUS treatment are predominantly those associated with laparoscopic surgery and those associated with irritation and/or treatable local infections at the site of the pocket for the pulse generator. The rechargeable TANTALUS II system has been well accepted in those patients now receiving it.

GCM treatment with the TANTALUS system promises to provide an innovative new tool that utilizes normal physiological pathways to improve management of patients with type 2 diabetes.
1. Baggio LL, Drucker DJ, Biology of incretins: GLP-1 and GIP, Gastroenterology, 2007;132:2131-7.

2. Buchwald $H$, Avidor $Y$, Braunwald $E$, et al., Bariatric surgery: $A$ systematic review and meta-analysis, JAMA, 2004;292:1724-37.

3. Moran TH, Gut peptide signaling in the controls of food intake, Obesity, 2006;14(Suppl. 5):250S-253S.

4. Young A, Amylin: Physiology and pharmacology, Adv in Pharmacology, 2005;52:281-8.

5. Rolls B, Roe LS, Meengs JS, Reductions in portion size and energy density of foods are additive and lead to sustained decreases in energy intake, Am J Clin Nutr, 2006;83:11-17.

6. Phillips RJ, Powley TJ, Gastric volume rather than nutrients content inhibit food intake, Am J Physiology, 1996;271:R766-9.

7. Jones KL, Doran SM, Hyeek, et al., Relation between postprandial satiation and antral area in normal subjects, Am I Clin Nutr, 1997; (66):127-32.

8. Ladabaum U, Koshy SS, Woods ML, et al., Differential symptomatic and electrogastrographic effects of distal and proximal human gastric distension, Am J Physiol, 1998:275:418-24.

9. Burkhoff $D$, Shemer I, Felzen B, et al., Electrical currents applied during a refractory period can modulate cardiac contraction in vitro and in vivo. Heart Fail Rev, 2001:6:27-34.

10. Peles S, Petersen J, Aviv R, et al., Enhancement of antral contractions and vagal afferent signaling with synchronized electrical stimulation, Am J Physiol Gastrointest Liver Physiol, 2003;285:G577-85.

11. Pochi A, Lam TK, Gutierrez-Juarez R, et al., Hypothalamic KATP channels control hepatic glucose production, Nature, 2005;434: 1026-31.

12. Pochi A, Obici S, Schwartz GJ, et al., A brain-liver circuit regulates glucose homeostasis, Cell Metab, 2005;1:53-61.

13. Bohdjalian A, Prager $G$, Aviv R, et al., One year experience with TantalusTM: a new surgical approach to treat morbid obesity, Obes Surg, 2006;16:627-34.

14. Pappone C, Vicedomini G, Salvati A, et al., Electrical modulation of cardiac contractility: clinical aspects in congestive heart failure, Heart Fail Rev, 2001:6:55-60.

15. Imai M, Rastogi S, Gupta RC, et al., Therapy with cardiac contractility modulation electrical signals improves left ventricular function and remodeling in dogs with chronic heart failure, J Am Coll Cardiol, 2007:49:2120-28.

16. Wang GJ, Yang J, Volkow ND, et al., Gastric stimulation in obese subjects activates the hippocampus and other regions involved in brain reward circuitry, Proc Nat Acad Sci USA, 2006;103:15641-5.

17. Zhu JN, Zhang YP, Song YN, et al., Cerebellar interpositus nuclear and gastric vagal afferents reach and converge onto glycemiasensitive neurons of the ventromedial hypothalamic nucleus in rats, Neurosci Res, 2004;4:405-17.

18. DeLuca M, Segato $G$, Busetto $L$, et al., Progress in implantable gastric stimulation: summary of results of the European multicenter study, Obes Surg, 2004;14(Suppl. 1):S33-S39.

19. Shikora $S A$, Implantable gastric stimulation for the treatment of severe obesity, Obes Surg, 2004;14:545-8.

20. Cigaini V, Long-term follow-up of gastric stimulation for obesity:the Mestre 8 year experience, Obes Surg, 2004;14(Suppl. 1):S14-\$22.

21. Cigaina $V$, Hirschberg $A$, Plasma ghrelin and gastric pacing in morbidly obese patients, Metab Clin \& Exper, 2007:56:1017-21.

22. Cigaini V, Hirschberg A, Gastric pacing for morbid obesity: plasma levels of gastrointestinal peptides and leptin, Obes Res, 2003;11: $1456-62$. 